\title{
ICTERÍCIA: DA MANIFESTAÇÃO AO MANEJO CLÍNICO-TERAPÊUTICO
}

\author{
Felipe Gaia de Sousa ${ }^{1}$ \\ Roberto Baracat de Araújo ${ }^{2}$
}

\begin{abstract}
RESUMO
A icterícia é considerada como a pigmentação amarelada de regiões corpóreas como esclera, pele e mucosas. É uma manifestação presente em variadas doenças e pode ser classificada em icterícia pré-hepática, hepática ou pós-hepática. A categorização dos tipos de icterícia e suas identificações são fundamentais para que haja um maior conhecimento acerca do seu local de ocorrência, da sua gravidade e progressão. A condição pode ser observada em cães e gatos, e cada tipo pode estar associado a doenças distintas como processos hemolíticos, doenças hepatocelulares, obstruções de fluxo biliar, dentre outros. O diagnóstico da manifestação pode ser determinado por meio dos dados coletados na anamnese, exame clínico e/ou complementar, este último mediante necessidade. O tratamento varia e depende da causa base que esteja associada como terapias medicamentosas a intervenções cirúrgicas.
\end{abstract}

Palavras-chave: icterícia, causa base, condição clínica, gravidade

\section{ICTERUS: FROM MANIFESTATION TO CLINICAL-THERAPEUTIC MANAGEMENT}

\begin{abstract}
Jaundice is considered the yellowish pigmentation of body regions such as the sclera, skin and mucous membranes. It is a manifestation present in several diseases and can be classified as pre-hepatic, hepatic or post-hepatic jaundice. The categorization of types of jaundice and their identification are essential for greater knowledge about its place of occurrence, its severity and progression. The condition can be observed in dogs and cats, and each type can be associated with different diseases such as hemolytic processes, hepatocellular diseases, bile flow obstructions, among others. The diagnosis of the manifestation can be determined through data collected through anamnesis, clinical and/or complementary examination, the latter as needed. Treatment varies and depends on the underlying cause that is associated with drug therapies and surgical interventions.
\end{abstract}

Keywords: jaundice, basic cause, clinical condition, gravity

\section{ICTERICIA: DE LA MANIFESTACIÓN AL MANEJO CLÍNICO-TERAPÉUTICO}

\section{RESÚMEN}

La ictericia se considera la pigmentación amarillenta de regiones del cuerpo como la esclerótica, la piel y las membranas mucosas. Es una manifestación presente en varias enfermedades y puede clasificarse en ictericia prehepática, hepática o poshepática. La categorización de los tipos de ictericia y su identificación son fundamentales para un mayor conocimiento sobre su lugar de aparición, su gravedad y progresión. La afección se puede observar en perros y gatos, y cada tipo puede asociarse con diferentes enfermedades como

\footnotetext{
${ }^{1}$ Pontifícia Universidade Católica de Minas Gerais/PUC Minas. Correspondência: fgaias@outlook.com.

${ }^{2}$ Universidade Federal de Minas Gerais - UFMG, baracat@vet.ufmg.br.
} 
procesos hemolíticos, enfermedades hepatocelulares, obstrucciones del flujo biliar, entre otras. El diagnóstico de la manifestación se puede determinar mediante la recogida de datos mediante anamnesis, exploración clínica y / o complementaria, esta última según sea necesario. El tratamiento varía y depende de la causa subyacente asociada con las terapias farmacológicas y las intervenciones quirúrgicas.

Palabras clave: ictericia, causa base, condición clínica, gravedad

\section{INTRODUÇÃO}

Os animais estão cada dia mais introduzidos no interior dos lares brasileiros e dessa forma, a preocupação de seus tutores com eles também eleva-se. Das diversas condições a que estão sujeitos, alterações na coloração usual de regiões como mucosas e pele, podem oferecer diversas hipóteses diagnósticas que merecem atenção. Destas, a icterícia é uma alteração importante sendo caracterizada pela presença de tons amarelados em regiões corpóreas e podendo estar associada a distintos diagnósticos. A condição pode ser classificada de três formas: pré-hepática, hepática e pós-hepática, e esta categorização permite ao clínico determinar possíveis distúrbios que estejam associados ao aparecimento da icterícia $(1,2)$. Dentre estes, cita-se: distúrbios hemolíticos, condições que induzem lesões hepatocelulares e quadros de obstrução do sistema hepatobiliar. É ideal que a causa base seja investigada e determinada para que seja possível determinar o melhor manejo terapêutico a ser instituído. $\mathrm{O}$ presente trabalho tem como objetivo descrever a ocorrência da icterícia, caracterizar e exemplificar os tipos mais comuns, além de abordar formas diagnósticas e de manejo clínicoterapêutico em geral.

\section{Icterícia}

\section{Conceito e mecanismos patofisiológicos}

Icterícia é uma manifestação clínica caracterizada pela tonalidade amarelada em superfícies corpóreas como: membranas revestidas por mucosas, região escleral e pele (2-4). A coloração amarela origina-se do excesso na concentração da bilirrubina, na região de tecidos e plasma, caracterizando-a como hiperbilirrubinemia. Para que esta seja visível, é necessária a detecção de concentrações do pigmento em torno de 35-50 $\mu \mathrm{mol} / \mathrm{L}$ (2-3 mg/dl), mas também quando ocorre incrementos de 5-10 vezes nos valores de referência para Sherding (1) e Webb (5). De acordo com Watson (2), "a icterícia é detectada em tecidos quando a concentração sérica for $\geq 2 \mathrm{mg} / \mathrm{dL}$ e no soro $\geq 1.5 \mathrm{mg} / \mathrm{dL}$ ". A manifestação da icterícia é um sinal que merece atenção por parte de tutores e profissionais veterinários, e segundo Zoran (6) "a icterícia clínica ocorre apenas nos casos mais graves, quando o fígado está acometido de modo difuso". Dessa forma, a condição pode-se estabelecer quando houver aumento na liberação, doenças de origem hepatocelular e/ou por retenções pigmentares $(1,6,7)$. A bilirrubina é obtida durante o processo de metabolização da hemoglobina.

A condição ictérica ocorre quando há um excesso que supera a condição de excreção pelo sistema hepatobiliar. A icterícia pode estar presente em diversas doenças como nas hemoparasitoses, hepatopatias, doenças imunomediadas, dentre outras. Categorizar a icterícia em sua ocorrência, localização e mecanismo causador pode auxiliar na determinação de qual região esteja com problema. Dessa forma, a icterícia é classificada em icterícia pré-hepática, hepática e pós-hepática, e diversas condições estão associadas a manifestação e ao aparecimento destes tipos $(1,2,5)$. A icterícia pré-hepática caracteriza-se pelo excesso de produção pigmentar ou por hemólise. A hepática é obtida por condições intrínsecas ao fígado 
como alterações hepatocelulares e/ou pelos impasses no processo de metabolismo da bilirrubina. A pós-hepática é observada quando há uma dificuldade na excreção das substâncias formadas (retenção), seja por dificuldade na condução da bilirrubina pelo sistema hepatobiliar ou por uma obstrução do fluxo biliar em região extra-hepática $(1,2,6)$.

\section{Metabolismo normal da bilirrubina}

A bilirrubina é a substância final do metabolismo da hemoglobina presente nas hemácias (2). Grande parte da hemoglobina (80\%) tem origem por meio das hemácias e o restante $(20 \%)$ por meio da quebra de outras porções proteicas que contém o grupamento heme, dentre as quais cita-se: a mioglobina (proteína de baixo peso molecular cuja finalidade é aumentar as concentrações de oxigênio tecidual para produção de energia); o complexo citocromo P450 (complexo enzimático composto por proteínas compostas pelo grupo heme); as peroxidases e catalases (proteínas responsáveis pela depuração de toxinas celulares) (1). As hemácias são produzidas por meio da linhagem eritrocitária presente na medula óssea. Após serem produzidas, são conduzidas à circulação para exercerem seu papel de carrear as moléculas de hemoglobina para tecidos, garantindo a oxigenação destes e evitando os quadros de hipóxia/anóxia. No entanto, desde que são produzidas e destinadas à circulação, elas apresentam um tempo de vida médio para cães de aproximadamente 120 dias e 70 para os gatos (8), e quando este estiver próximo, elas são induzidas a se autodestruírem sendo substituídas por novas hemácias $(2,9)$.

Ao final da meia vida, elas passam por uma modificação metabólica necessária, a qual induz alterações de funcionalidade. Dessa forma, a membrana celular torna-se mais fraca e passível de rupturas. Com o prazo de meia vida esgotando-se e em associação com a condução das hemácias para regiões estreitas (ex. porções periféricas), elas se rompem. Além da destruição delas na circulação, o baço contribui com o processo de hemocaterese. Diante disso, os vasos sanguíneos tornam-se compostos de novas hemácias e à medida que estas tornam-se afuncionais são destruídas pelo baço ou induzidas a apoptose (10). Após a destruição das hemácias, há liberação de moléculas de hemoglobina no sangue, as quais ficam soltas e circulantes $(1,10)$. Neste momento, o sistema mononuclear fagocitário (SMF) induz a fagocitose da hemoglobina por macrófagos a nível esplênico e medular, e por células de Kupffer em parênquima hepático (2). Ocorre a captação da hemoglobina pelo SMF, a qual é convertida em três substâncias: ferro livre, monóxido de carbono e biliverdina, devido à enzima heme-oxigenase (HO-1) ativada por estresse. A biliverdina é convertida em bilirrubina livre por meio da biliverdina - redutase e alcança o plasma (Figura 1) $(1,2,10)$.

A produção diária de bilirrubina é variável, com valores médios entre $3-5 \mathrm{mg} / \mathrm{kg}(10,12)$. De acordo com Martelli (10) "essa bilirrubina é lipossolúvel e apolar, podendo ligar-se à albumina e sua fração livre atravessar a barreira hematoencefálica". Uma vez ligada à albumina, é fortemente direcionada ao fígado para ser metabolizada, e dessa forma ocorre o fim da fase primária ou pré-hepática da bilirrubina (1). Para que a segunda fase ocorra, ou etapa hepática, será necessário o auxílio de processos importantes: a captação (recolhimento da bilirrubina pelo fígado), a conjugação (ligação por meio de enzimas microssomais) e a excreção (liberação da substância no sistema hepatobiliar) (5).

No início do processo, a bilirrubina liga-se a proteínas plasmáticas, especialmente a albumina, tornando-se bilirrubina ligada à albumina $(3,9)$. Pela capacidade de percorrer toda a circulação e fluidos de natureza intersticial, ela é conduzida ao fígado. Esta bilirrubina também é conhecida como bilirrubina livre, não conjugada ou indireta devido à não ocorrência da conjugação por meio das enzimas de origem microssomal (1). O processo de captação é o recolhimento da bilirrubina livre pelo fígado em que a ligação com a albumina é rompida na região dos hepatócitos (dissociação), especialmente no local de contato entre as 
células hepáticas e os capilares sinusoides (2). Por meio de sistemas transportadores membranosos de natureza proteica, a bilirrubina é captada e torna-se disponível para outras etapas metabólicas $(1,10)$. De acordo com Sherding (1), "a capacidade de captação dos hepatócitos excede em muito a capacidade excretora".

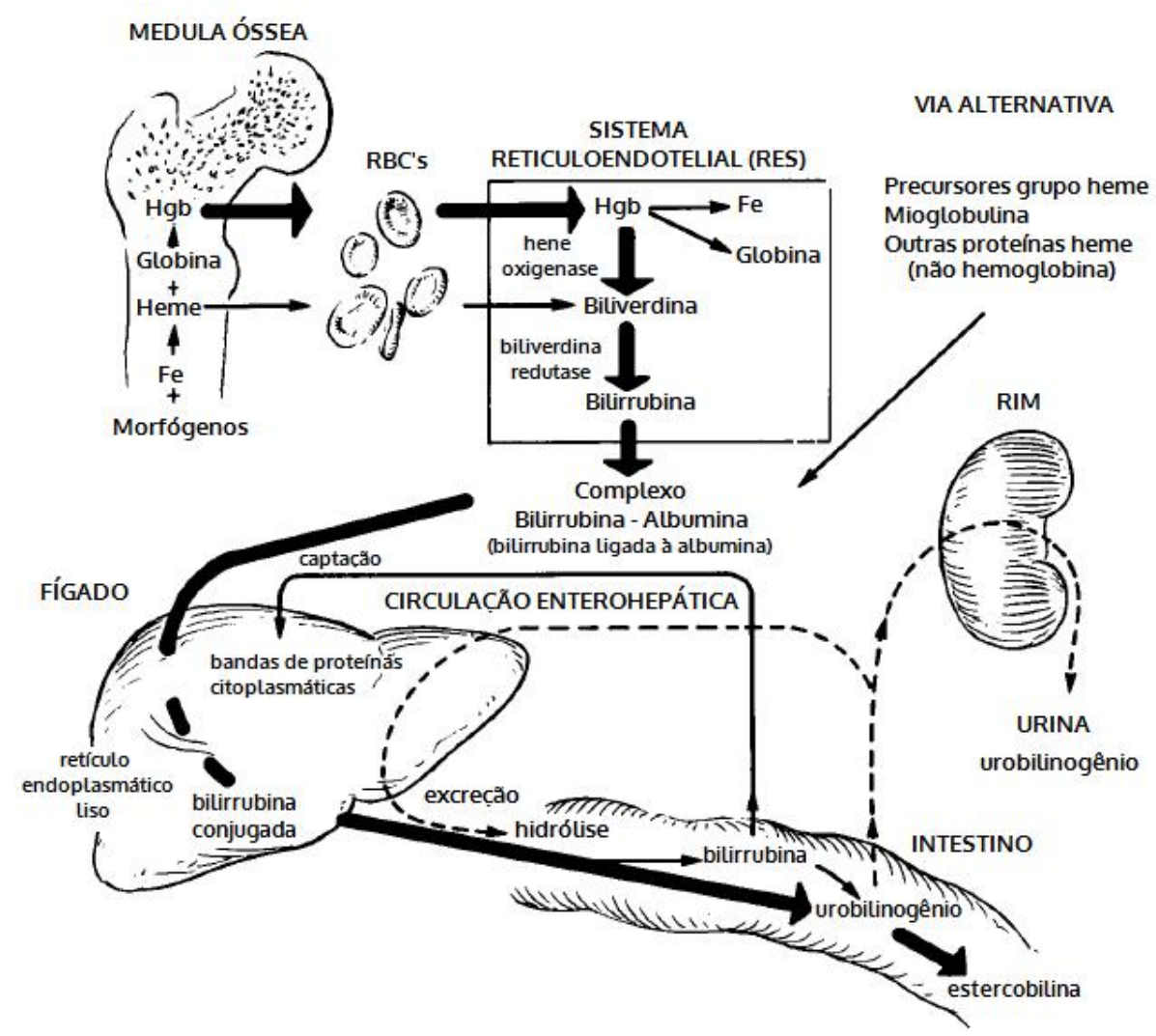

Figura 1. Metabolismo normal da bilirrubina pela quebra da hemoglobina liberada por meio da quebra das hemácias. Nota-se que a bilirrubina obtida é metabolizada e convertida em diversos produtos, sendo posteriormente eliminada via fezes. Adaptado de Gartner e Hollander (11).

No interior do hepatócito, a bilirrubina entra e liga-se a ligandina (processo de regulação do recolhimento de bilirrubina) para evitar o escoamento para a região do plasma (13). Diante disso, ocorre a ligação da forma não conjugada com o ácido glicurônico processo de conjugação da bilirrubina (9) e, mediante o estímulo da enzima UDP-glicuronil transferase, transforma-se na forma conjugada (forma polar e hidrossolúvel) (1). A bilirrubina conjugada, ou direta, é conduzida para a região biliar, em interface com a porção canalicular biliar. Segundo Martelli (10), certas regiões também podem exercer a função de conjugar a bilirrubina, embora em menor número, como a região tubular renal e intestinal de cães, embora não apresente evidências da função para gatos (1).

A bilirrubina conjugada é conduzida pelo sistema hepatobiliar até desembocar na região duodenal sendo eliminada com a bile (2). No entanto, a bilirrubina conjugada apresenta dificuldades de absorção e por isso é conduzida para o intestino grosso. A microbiota colônica existente (glicuronidases bacterianas) induz a transformação da bilirrubina conjugada em urobilinogênio (produto final). No entanto, uma pequena parcela dessa bilirrubina é reabsorvida pelas células intestinais (processo de desconjugação) por ação bacteriana intestinal e conduzida ao fígado $(1,2,10)$. O urobilinogênio pode ser eliminado de forma, intestinal e renal, embora grande parte seja via fezes (1). A excreção intestinal só é possível graças a oxidação, a qual transforma-o em estercobilina, permitindo que a substância seja 
removida do organismo pela matéria fecal (2). O restante do urobilinogênio retorna à circulação entero-hepática (10-20\%) (9) para sofrer uma nova metabolização e do pigmento que é absorvido, 1-5\% é eliminado por via urinária (Figura 1) (1).

\section{Icterícia pré-hepática, hepática e pós-hepática}

A icterícia pré-hepática é uma disfunção de acordo com Sherding (1) originada da "superprodução de bilirrubina resultante da hemólise". Por meio da intensa quebra eritrocitária, o excesso de bilirrubina é produzido por meio da liberação do grupo heme na circulação (2). Dessa forma, constata-se hiperbilirrubinemia, a qual pode desenvolver-se em icterícia quando houver uma sobrecarga na condição de funcionamento e metabolismo pigmentar hepático (2). Assim, o fígado não desempenha as etapas para captar, conjugar e excretar os produtos do metabolismo da substância (1) (Figura 2).

A
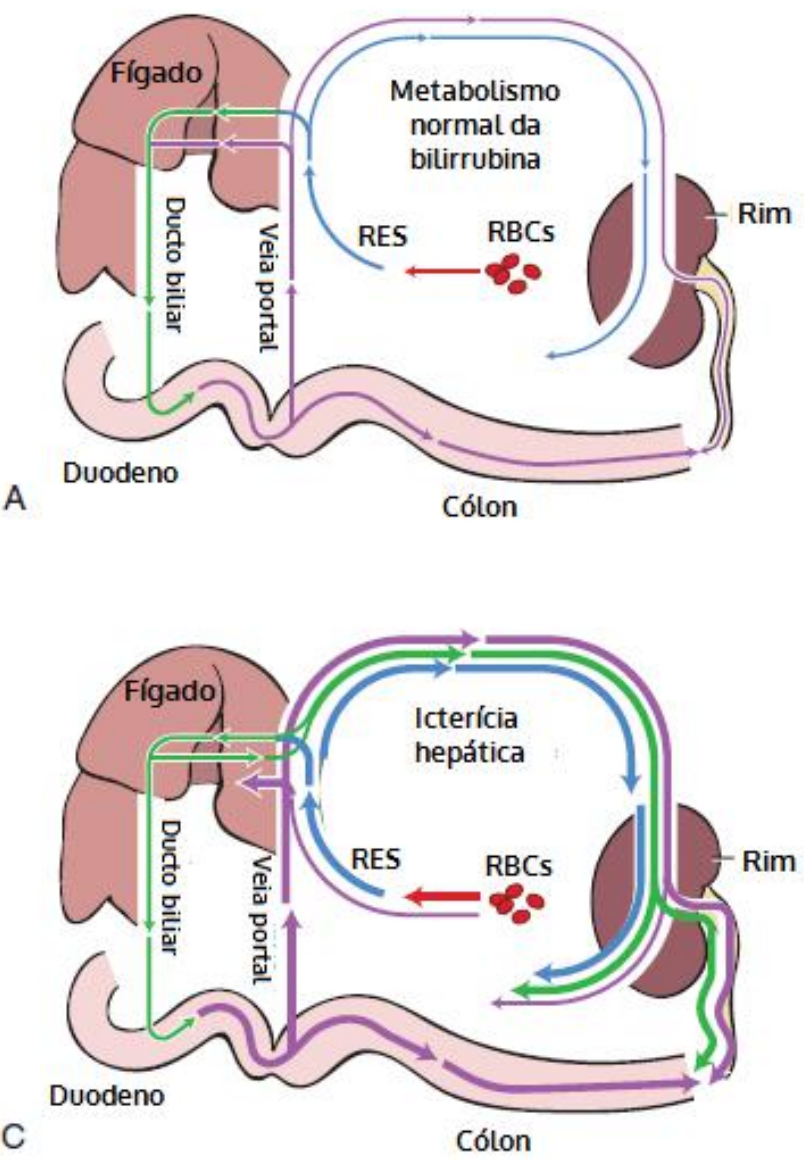

B

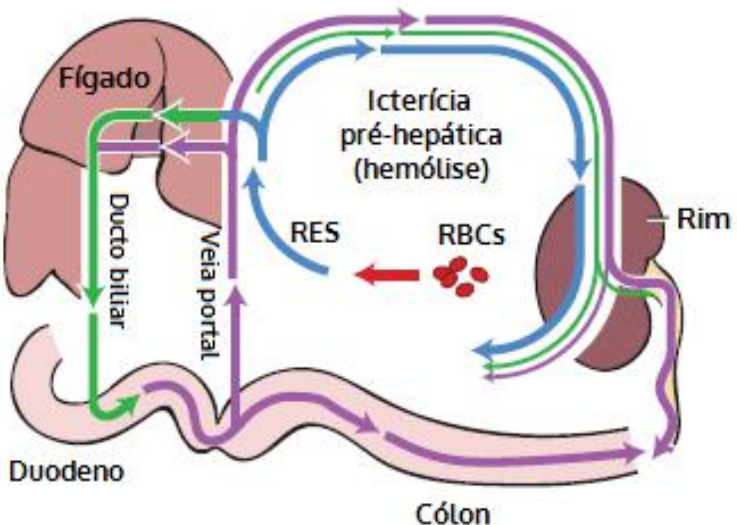

$\mathrm{D}$

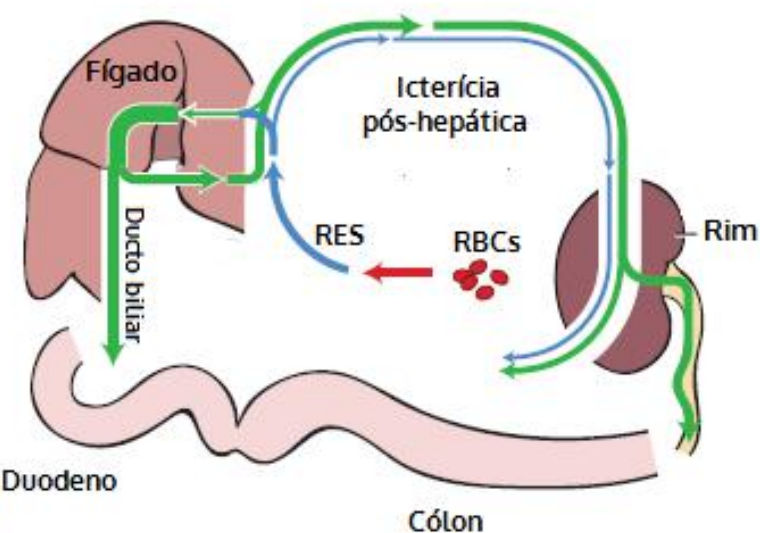

Figura 2. Esquema do metabolismo normal da bilirrubina (A) e a manifestação dos tipos de icterícia descritos (pré-hepática $-\mathrm{B}$, hepática $-\mathrm{C}$ e pós-hepática $-\mathrm{D}$ ). $\mathrm{O}$ funcionamento normal baseia-se na quebra da hemoglobina, produção pigmentos (captação, conjugação e excreção). Por meio dessa condição, mecanismos que induzem a hemólise, alterações hepatocelulares e obstruções da via de saída tendem a proporcionar aumentos na produção, redução na metabolização e dificuldades para excreção, com possível acúmulo pigmentar em superfícies corpóreas. Considere: RBCs - hemácias; RES - sistema mononuclear fagocitário. Adaptado de Sherding (1). 
Nota-se que a bilirrubina que retorna do fígado é direcionada para a grande circulação, promovendo o tingimento das superfícies corpóreas. Para que haja um excesso produtivo da bilirrubina é necessário que a hemólise ocorra de forma abrupta e intensa, sendo capaz de promover a sobrecarga funcional hepática. A miscelânea dos tipos de bilirrubina (indireta e direta) pode ser observada através da hemólise e da colestase intra-hepática (observada em pacientes com complicações hepáticas hipoxêmicas). Além disso, o aumento de bilirrubina conjugada no intestino induz maiores produções de urobilinogênio, com repercussão fecal (aumento de estercobilina) e urinária (aumento de urobilinogênio), e alterações de coloração (tom laranja amarronzado) (1) (Figura 2).

A condição de icterícia hepática refere-se a situações em que há colestase intra-hepática com alterações parenquimatosas importantes como: lesões de hepatócitos, necrose ou disfunções hepáticas $(1,2)$. Este tipo de icterícia desenvolve-se devido às consequências das alterações de parênquima que induzem em impasses principalmente de metabolização e condução da bilirrubina através das vias biliares da região intra-hepática (1,3) (Figura 2). A progressão da icterícia depende do local em que as lesões estejam, de forma que, quanto mais próximas a região portal ou em estruturas adjacentes, piores serão as consequências devido à condução biliar centrífuga (12). Watson (2) afirma que "é mais provável que a icterícia seja uma característica clínica se o distúrbio hepático envolver principalmente a zona 1 do que a 3". A porção não conjugada também sofre o retorno à circulação assim como a conjugada devido ao excesso de bilirrubina no interior dos hepatócitos (conjugação pigmentar dificultada). Os hepatócitos podem ainda apresentar lesões agravadas por condições que associem hepatopatias com acúmulo dos ácidos biliares. Ressalta-se que uma pequena porção da bilirrubina conjugada e do urobilinogênio são eliminados por via urinária (1) (Figura 2).

Quadros de icterícia pós-hepática associam-se com situações que promovam obstrução da via de saída hepatobiliar $(3,5)$, principalmente por alterações nas vias biliares de região extra-hepática (represamento do material). Considera-se que as icterícias pós-hepáticas são caracterizadas pela presença das duas formas de bilirrubina, com abundância do tipo conjugada. O padrão misto observado é derivado da colestase extra-hepática imposta pela obstrução (refluxo da bilirrubina para a grande circulação) e pelo represamento biliar no parênquima hepático (lesão de células e disfunções no processo conjugador). Salienta-se que uma pequena porção conjugada pode ser eliminada pela urina, embora não seja possível determinar a presença de urobilinogênio pela obstrução na liberação do precursor para a região intestinal. Além disso, fezes de animais portadores dessa condição apresentam-se de cor clara pela não liberação de bile na região duodenal (1) (Figura 2).

\section{Diagnóstico da icterícia}

\section{Avaliação clínica e laboratorial do paciente}

Determinar a presença de icterícia não é algo difícil, e por ser uma alteração visível, tanto o médico veterinário quanto o tutor podem constatar. Ressalta-se que fatores externos como a luminosidade ambiental podem interferir na observação por parte do observador e possibilitar a ocorrência de falsos positivos (1). No entanto, é primordial que a causa base para a manifestação seja estudada e determinada (2). Além disso, determinar qual o tipo de icterícia que esteja ocorrendo, pode fornecer informações valiosas a respeito de possíveis condições que possam estar envolvidas e para isso, o máximo de dados coletados com o tutor e pelo exame físico será importante.

A depender da (s) doença (s) que for (em) suspeitada (s), diversas opções diagnósticas estão disponíveis como hemograma, bioquímico, urinálise, testes rápidos, ultrassonografias, citologias hepáticas, dentre outras. Ressalta-se que a manifestação poderá ter apresentação 
leve a grave devido à causa de origem e ao grau de hiperbilirrubinemia $(2,4)$. É fundamental que todos os possíveis diferenciais sejam levantados e excluídos a depender das condições observadas durante o exame físico do paciente (Figura 3). Inicialmente os tutores procuram auxílio após notarem alterações na cor de pele e mucosas, urina e fezes. A urina pode apresentar cores que variam de verde a avermelhado (1) e as fezes, laranja a cinza claro (2). Além disso, sinais inespecíficos podem estar presentes como apatia, prostração, vômitos, diarreias, perdas de peso, dentre outros. É necessário que a anamnese e o exame clínico desses animais sejam feitos de forma detalhada, em busca de maiores informações que possam eliminar ou confirmar suspeitas como: ingestão de toxinas, presença de agentes infecciosos, uso de medicamentos hepatotóxicos, dentre outros. Particularidades entre espécies devem ser avaliadas antes e após a realização de exames como a capacidade de reabsorção tubular (2).

Laboratorialmente, dados valiosos podem ser obtidos e devem ser utilizados de forma conjunta com achados de anamnese e exame físico. No hemograma, pode-se observar hemólise com redução da contagem global de hemácias e alterações nas cores do plasma ou soro sanguíneo. A pseudohiperbilirrubinemia pode ser erroneamente interpretada devido à lipemia (2). Salienta-se que demais causas de hemólise devem ser investigadas quando o hematócrito estiver menor que $20 \%$ e com sinais de regeneração (1). No bioquímico, observase hiperbilirrubinemia quando os valores obtidos estão acima de $2-3 \mathrm{mg} / \mathrm{dl}(35-50 \mu \mathrm{mol} / \mathrm{L})$, sendo uma alteração observada antes da presença da cor amarelada (1). De acordo com Watson (2) "a maioria das pesquisas publicadas concordam que concentrações acima de 0,3 $\mathrm{mg} / \mathrm{dL}$ em gatos e $0,6 \mathrm{mg} / \mathrm{dL}$ em cães são anormais.

As bilirrubinas podem apresentar aumentos concomitantes ou isolados nas concentrações plasmáticas (Quadro1). A bilirrubina livre poderá estar aumentada em hemólises, hemorragias, transfusões, resolução de hematomas, entre outros. Incrementos de bilirrubina conjugada estão associadas com disfunções no funcionamento hepático por variadas causas como: agentes infecciosos, toxicidades e/ou alterações da via hepatobiliar. No entanto, o aumento das duas bilirrubinas pode ocorrer em situações como: alterações de funcionalidade celular hepática, hemólise intensa e obstruções hepatobiliares (9). A urinálise, pode ser capaz de detectar bilirrubinúria e concentrações elevadas de urobilinogênio, embora a bilirrubina seja alterável por fatores externos como luz e temperatura (1). Além disso, demais indicadores deverão ser investigados apenas em caso de necessidade. Bilirrubinúria é um achado comum em cães com valores de densidade $(>1,025)$ devido à baixa capacidade reabsortiva tubular, porém em gatos a condição absortiva é maior com maior excreção urinária e associada a doença (2).

Quadro 1. Descrição das alterações observadas na urina, fezes e sangue de pacientes portadores dos três tipos de icterícia. Anatolievna (4).

\begin{tabular}{lcccccc}
\hline & \multicolumn{2}{c}{ Urina } & \multicolumn{2}{c}{ Fezes } & Sangue \\
\cline { 2 - 6 } $\begin{array}{l}\text { Tipos de } \\
\text { icterícia }\end{array}$ & Bilirrubina & Urobilinogênio & Estercobilina & $\begin{array}{c}\text { Bilirrubina } \\
\text { direta }\end{array}$ & $\begin{array}{c}\text { Bilirrubina } \\
\text { indireta }\end{array}$ & $\begin{array}{c}\text { Bilirrubina } \\
\text { direta/total }\end{array}$ \\
\hline $\begin{array}{l}\text { Pré- } \\
\text { hepática }\end{array}$ & 0 & $\downarrow$ ou N & $\uparrow$ & $\mathrm{N}$ & $\uparrow$ & 0,2 \\
\hline Hepática & + & $\uparrow$ & $\uparrow, 0$ ou N & $\uparrow$ & $\uparrow$ & $0,2-0,7$ \\
\hline $\begin{array}{l}\text { Pós- } \\
\text { hepática }\end{array}$ & + & $\downarrow$ ou N & $\downarrow$ & $\uparrow$ & $\uparrow$ & 0,5 \\
\hline
\end{tabular}

\section{Doenças em que há manifestação da condição de icterícia}

Diversas doenças podem apresentar manifestação clínica de icterícia evidente. É necessário realizar a exclusão dos diferenciais por meio dos sinais observados. Ressalta-se 
que a origem da condição deve ser determinada pois as causas que induzem as icterícias préhepáticas, hepáticas e pós-hepáticas são distintas entre si. Mediante a apresentação clínica em associação com os dados obtidos, é possível direcionar a conduta diagnóstica e terapêutica (3).

\section{Icterícia pré-hepática}

As doenças que cursam com icterícia pré-hepática são aquelas em que a hemólise esteja associada, como: hemoparasitoses e agentes infecciosos como a leptospirose (discutida na seção hepática), doenças imunomediadas, uso de toxinas e drogas, quadros de envenenamento, distúrbios eletrolíticos, condições hereditárias, fragmentações eritrocitárias periféricas, adversidades durante a terapia transfusional, isoeritrólise em neonatos, dentre outros (1).

Os hemoprotozoários como Babesia spp. $(14,15)$, Mycoplasma spp. $(5,16)$, Cytauxzoon felis $(5,17)$ adentram as hemácias e induzem a quebra eritrocitária com liberação de hemoglobina na circulação (1). Dentre as doenças imunomediadas, a anemia hemolítica é considerada como um exemplo clássico devido à destruição maciça de eritrócitos - reação de hipersensibilidade do tipo II $(18,19)$. O vírus da imodeficiência felina - FIV e o vírus da leucemia felina - FeLV também estão associados a icterícia devido à imunodepressão presente $(4,5)$.

$\mathrm{O}$ uso de toxinas e fármacos como acetaminofeno (formação de metahemoglobina e sobrecarga hepática) (20) e vitamina K (anticoagulante) induzem quadros de hemólise. Envenenamentos por serpentes (ex. Bothrops sp.) podem induzir a destruição eritrocitária devido à ação hemorrágica presente no veneno (21). A hipofosfatemia pode promover hemólise pela redução da síntese de 2,3-difosfoglicerato e trifosfato de adenosina - ATP (22). Terapias transfusionais induzem hemólise intravascular quando houver reação do tipo II ao sangue transfusionado (23). As demais condições não são frequentes, embora possam causar destruição eritrocitária.

\section{Icterícia hepática}

As condições associadas com icterícia hepática estão relacionadas ao acometimento do parênquima hepático como reações farmacológicas por fármacos tóxicos, doenças infecciosas e hepáticas, lesões crônicas, colestases intra-hepáticas, neoplasias, retenções de gordura, entre outras (1). O uso e/ou contato com drogas e toxinas hepatotóxicas estão relacionadas com lesões diretas após o início da administração como micotoxinas, anti-inflamatórios não esteroidais, antiparasitários, anticonvulsivantes, dentre outros, além do contato com substâncias químicas (2).

As doenças infecciosas como a hepatite infecciosa causada pelo Adenovírus canino tipo-1 induzem a necrose do parênquima lobular hepático, fibrose e disfunções funcionais $(24,25)$. A leptospirose é uma importante zoonose causada por bactérias do gênero Leptospira spp. e proporcionam lesão hepática agudizada com manifestação ictérica $(3,26)$. A PIF causada pelo coronavírus felino também induz a ocorrência de icterícia por vasculites (forma efusiva), induzindo o aumento da permeabilidade vascular e extravasamento de fluidos (27). Quadros de colangites e colangiohepatites estão associados com inflamações das vias biliares (especialmente o ducto biliar) e do parênquima hepático (28) induzindo alterações funcionais (29).

A sepse pode promover colestase hepática e manifestação de icterícia (agravo do quadro) devido à redução da perfusão, congestão e/ou lesões celulares com repercussão funcional $(30,31)$. A lipidose hepática felina é outra condição que propicia a icterícia devido ao acúmulo de gordura intra-hepática, dificultando o processo de metabolização e funcionamento do fígado (4,6,32). Além disso, neoplasias e/ou processos metastáticos hepáticos também 
induzem disfunções funcionais (1). No geral, qualquer condição que cause lesão ou necrose do parênquima hepático com redução da capacidade metabólica e funcional podem induzir ao aparecimento de icterícia hepática (1).

\section{Icterícia pós-hepática}

No caso das doenças que estão associadas com icterícia pós-hepática, a maioria relaciona-se com alterações do sistema hepatobiliar como doenças gastroentéricas, rupturas biliares, neoplasias, verminoses, condições inflamatórias, além de processos colestáticos. A pancreatite é caracterizada pela inflamação aguda ou crônica do parênquima hepático, com ou sem necrose, a qual pode induzir ao aparecimento de icterícia devido à gravidade da doença por acometimento de órgãos adjacentes $(28,33,34)$ e obstrução biliar extra-hepática (2).

As obstruções e doenças das vias biliares como colelitíases, colédocolitíases, inflamações ductais, impõe dificuldades e/ou impedimentos das vias de saída dos produtos do metabolismo da bilirrubina, permitindo com que haja o represamento e desenvolvimento da condição $(1,35)$. Dentre as verminoses cita-se as platinossomíases por Platynosomum sp., as quais são responsáveis por obstruir o fluxo de liberação ductal biliar (represamento dos produtos) (36). De forma geral, qualquer condição que impeça e/ou dificulte a liberação e a condução dos produtos do sistema hepatobiliar podem induzir ao aparecimento de icterícia pós-hepática $(2,3)$.

Andrade et al. (3) realizaram um estudo por meio da necropsia de 83 cães ictéricos entre o período de 2014-2017, com o intuito de determinar e classificar as causas de icterícia na espécie. Os animais foram segregados em 4 grupos: 24 cães com pré-icterícia, 45 com icterícia hepática, 13 com icterícia pré e hepática e $1 \mathrm{com}$ pós-hepática. Diversas causas foram observadas e distribuídas da seguinte forma: 51/83 causas infecciosas, 13/83 neoplasias, 11/83 degenerações, 6/83 disfunções crônicas e 1/83 obstruções. Dentre aos agentes infecciosos, os autores observaram leptospirose, hemoparasitoses e condições que sugeriram septicemia, confirmando o papel destes na ocorrência de icterícia.

\section{Outras ferramentas diagnósticas}

Em determinadas ocasiões poderá ser necessário a utilização de algumas opções diagnósticas como a ultrassonografia, radiografias, tomografias e em alguns casos biópsias hepáticas (4). Salienta-se que a escolha da realização de tais exames deve ser determinada apenas em casos de necessidade. Dependendo do que for obtido na anamnese e exame clínico, essas ferramentas oferecem grandes informações para acompanhamento da condição apresentada, confirmação e/ou descarte de suspeitas diagnósticas, avaliação de medidas terapêuticas, dentre outras (1).

\section{Princípios terapêuticos gerais}

A terapêutica desenvolvida para tratamento da icterícia deve ser baseada na determinação da causa base e dessa forma, não existe protocolo terapêutico definido. Cada terapia é adaptada e ajustada para cada paciente, baseando-se nas informações obtidas por exames físico, laboratorial e complementares, se necessário. Segundo Sherding (1) "em geral, causas de icterícia pré-hepática e hepática são tratadas com medicamentos, enquanto que algumas formas de icterícia pós-hepática podem requerer intervenção cirúrgica".

Situações que estejam associadas com icterícia pré-hepática podem demandar terapia imunossupressora com uso de corticoides e demais drogas imunossupressoras como azatioprina, ciclosporinas, micofelato mofetil, uso de antioxidantes, redução da exposição a 
toxinas e uso de fármacos, terapias antimicrobianas, entre outros (37). Quadros ictéricos hepáticos e pós-hepáticos podem demandar terapia medicamentosa como o uso de ácido ursodesoxicólico $\left(\mathrm{Ursacol}^{\circledR}\right)$ e S-adenosil- L-metionina $\left(\mathrm{SAMe}^{\circledR}\right)$, além de suporte nutricional específico (6). No entanto, dependendo da gravidade e da condição apresentada, o intervencionismo cirúrgico pode ser de grande valia como por exemplo o uso de stents (38), colecistoenterostomias $(1)$, colecistectomias $(39,40)$, entre outras.

\section{CONSIDERAÇÕES FINAIS}

A icterícia é uma alteração importante e não deve ser negligenciada por tutores e médicos veterinários. A condição está associada a diversas doenças e investigar a fundo os motivos que ocasionaram no aparecimento da condição é de grande valia. Além disso, determinar qual o tipo da icterícia pode contribuir para o levantamento de suspeitas diagnósticas, uma vez que fatores externos e internos ao fígado podem corroborar para a manifestação. Todo paciente que estiver apresentando membranas mucoides e pele de coloração amarelada deve passar por avaliações criteriosas para determinação da causa base e do manejo terapêutico a ser instituído. Caso sejam necessários, exames diagnósticos complementares devem ser associados com os dados obtidos na anamnese e exame físico do paciente. Dessa forma, será possível conduzir o caso, avaliar a progressão e a terapêutica estabelecida para a resolução da condição.

\section{REFERÊNCIAS}

1. Sherding RG. Icterus. In: Washabau RJ, Day MJ, editors. Canine and feline gastroenterology. St. Louis: Elsevier Saunders; 2013. p. 140-7.

2. Watson PJ. Clinical manifestations of hepatobiliary and pancreatic disease. In: Nelson RW, Couto CG, editors. Small animal internal medicine. 6th ed. St. Louis: Elsevier; 2020. Chap. 33 , p. 518-30.

3. Andrade MC, Oliveira LB, Santos AF, Moreira MVL, Pierezan F, Ecco R. Differential diagnoses in 83 dogs with icterus. Pesqui Vet Bras. 2020;40(6):451-65. doi: 10.1590/16785150-PVB-6482.

4. Anatolievna KO. Diagnostic tests of jaundice in cats and dogs. Interconf [Internet]. 2021 [citado 10 Maio 2021];(57):1-9. Disponível em: https://ojs.ukrlogos.in.ua/index.php/interconf/article/view/12717

5. Webb CB. The yellow cat: diagnostic \& therapeutic strategies. Todays Vet Pract [Internet]. 2016 [citado 10 Maio 2021];6(5):38-50. Disponível em: https://todaysveterinarypractice.com/the-yellow-cat-diagnostic-therapeutic-strategiescearticle/

6. Zoran DS. Diseases of the liver. In: Little SE, editor. The cat - clinical medicine and management. St. Louis: Saunders; 2012. p. 522-30.

7. Ware WA, Ward JL. Clinical manifestations of cardiac disease. In: Nelson RW, Couto CG, editors. Small animal internal medicine. 6th ed. St. Louis: Elsevier; 2020. Chap. 1, p. 1-12. 
8. Duncan JR, Prasse KW. Patologia clínica veterinária. Rio de Janeiro: Guanabara Koogan; 1982.

9. González FHD, Silva SC, editores. Patologia clínica veterinária: texto introdutório. Porto Alegre: Universidade Federal do Rio Grande Do Sul; 2008.

10. Martelli A. Síntese e metabolismo da bilirrubina e fisiopatologia da hiperbilirrubinemia associados à Síndrome de Gilbert: revisão de literatura. Rev Med Minas Gerais. 2012;22(2):216-20.

11. Gartner LM, Hollander M. Disorders of bilirubin metabolism. In: Assali N, editor. Pathophysiology of gestation. 3th ed. New York: Academic Press; 1972. p. 455-503.

12. Center SA. Fisiologia, diagnóstico laboratorial, e afecções do fígado. In: Ettinger SJ, Feldman EC, editores. Tratado de medicina interna veterinária: moléstias do cão e do gato. 4a ed. São Paulo: Manole; 1996. Cap. 106, p. 1745-817.

13. Martinelli ALC. Jaundice. Medicina (Ribeirão Preto). 2004;37(3/4):p.246-252. doi: 10.11606/issn.2176-7262.v37i3/4p246-252.

14. Dias VACM, Ferreira FLA. Canine babesiosis: review. PUBVET. 2016;10(12):886-8. doi: 10.22256/pubvet.v10n12.886-888.

15. Reddy BS, Sivajothi S, Varaprasad Reddy LSS, Solmon Raju KG. Clinical and laboratory findings of Babesia infection in dogs. J Parasit Dis [Internet]. 2016 [citado 13 Maio 2021];40(2):268-72. Disponível em: https://pubmed.ncbi.nlm.nih.gov/27413291/

16. Ferraz A, Barwaldt ET, Pires BS, Lima CM, Bierhals ES, Nobre MO, et al. Mycoplasmosis in feline domestic, FeLV (+), Case Report. Vet Zootec. 2020;27:1-7. doi: 10.35172/rvz.2020.v27.504.

17. Ribeiro TMP, Santos HD, Reis TS, Sousa SAP, Furquim MEC, André MR, et al. Infection by Cytauxzoon spp. in domestic felines. Med Vet (UFRPE). 2019;13(3):362-74. doi: https://doi.org/10.26605/medvet-v13n3-3312.

18. Ramos LT, Leite AKRM. Alterações clínicas e laboratoriais em um cão com anemia hemolitica imunomediada: relato de caso. Rev Cient Eletronica Med Vet. 2017;28:1-10.

19. Gorenstein TG, Portilho FVR, Calobrizi NP, Gonçalves DS, Paes AC, Takahira RK. Thromboelastometry assessment of mycophenolate mofetil effect on thrombotic risk in dogs with primary and secondary immune-mediated hemolytic anemia and by Erhlichia canis infection. Arq Bras Med Vet Zootec [Internet]. 2021 [citado 20 Maio 2021];73(2):335-42. Disponível em: https://www.scielo.br/j/abmvz/a/jVmhCmmZ9qYBDTGbDB6wy8G/abstract/?lang=en

20. Dorigon O, Almeida ACVR, Costa FVA. Acetaminophen toxicity in cats. Rev Cienc Agrovet. 2013;12(1):88-93. 
21. Alves LMC, Pereira JA, Barreto CLMS, Figueiredo KBW, Morante NJ. Diagnóstico e tratamento de acidente ofídico por serpente do gênero Bothrops em cão. Vet Not. 2020;26(1):22-31. doi: https://doi.org/10.14393/VTN-v26n1-2020-51930.

22. Silva RD. Avaliação dos distúrbios ácido-base e eletrolíticos de cães com cetose e cetoacidose diabética [dissertação]. São Paulo: Faculdade de Medicina Veterinária e Zootecnia, Universidade de São Paulo; 2006.

23. Maia J, Sousa AV, Raquel RM. Importância dos cuidados de enfermagem na transfusão de cães e gatos. Cienc Vet UniFil [Internet]. 2018 [citado 10 Maio 2021];1(3):66-8. Disponível em: http://periodicos.unifil.br/index.php/revista-vet/article/view/985

24. Souto EPF, Maia LA, Ferreira JS, Gomes LCVM, Carneiro RS, Driemer D, et al. Epidemiological, clinical and pathological aspects of infectious canine hepatitis: 15 cases. Pesqui Vet Bras. 2018;38(8)1608-14. doi: 10.1590/1678-5150-PVB-5468.

25. Gonçalves GS, Mendonça MO, Jané DR, Franco IG, Souza FB. Infeccious canine hepatitis: a review about physiopathology and anatomopathologicals aspects of the disease. Rev Cient Eletronica Med Vet. 2020;34:1-8.

26. Silva RA, Rodrigues MC, Santana MV, Rodrigues KF, Sousa FB, Silva TS, et al. Leptospirose canina: relato de caso. PUBVET. 2018;12(6):1-6. doi: 10.22256/pubvet.v12n6a102.1-6.

27. Massitel IL, Viana DB, Ferrante M. Peritonite infecciosa felina: revisão. PUBVET. 2021;15(1):1-8. doi: https://doi.org/10.31533/pubvet.v15n01a740.1-8.

28. Černá P, Kilpatrick S, Gunn-Moore DA. Feline comorbidities - What do we really know about feline triaditis? J Feline Med Surg. 2020;22(11):1047-67. doi: https://doi.org/10.1177/1098612X20965831.

29. Coelho HE, Carvalho TF, Alberto H, Locce CC, Silva KDC, Barbosa CHG, et al. Case of biliary cirrhosis in feline Felis domesticus. PUBVET. 2011;5(37):1-14.

30. Gonzaga BC. Sepse em pequenos animais, revisão bibliográfica com ênfase em alterações cardíacas [dissertação]. Brasília: Faculdade de Agronomia e Medicina Veterinária, Universidade de Brasília, 2011.

31. Chacar FC, Guimarães-Okamoto PT, Melchert A, Arruda VK, Bento DD, Costa DC. Sepse em felinos. Vet Zootec. 2014;21(1):64-76, 2014.

32. Lima LTR, Araújo EKD, Silva MG, Honório TGAF, Costa SDP, Rodrigues KF, et al. Lipidose hepática secundaria à Colangiohepatite em felino doméstico sem raça definida: relato de caso. PUBVET. 2017;11(5):476-81. doi: http://dx.doi.org/10.22256/PUBVET.V11N5.476.

33. Mack IC. Pancreatitis in small animal medical clinic: a literature review. Rev Multidiscip Psicol. 2020;14(50):854-63. doi: https://doi.org/10.14295/idonline.v14i50.2479. 
34. Sousa FG, Rabelo AL, Rodrigues AK, Silva DET, Diniz GHS, Nunes ISS, et al. Canine pancreatitis: the danger in the routine of veterinarians: review. PUBVET. 2021;15(3):1-9. doi: 10.31533/pubvet.v15n03a769.1-9.

35. Center SA. Diseases of the gallbladder and biliary tree. Vet Clin North Am Small Anim Pract. 2009;39(3):543-598. doi: 10.1016/j.cvsm.2009.01.004.

36. Campos NC, Siqueira DF, Perin LR, Oliveira LC, Campos DR, Martins IVF. Natural infection by Platynosomum fastosum in domestic feline in Alegre, Espírito Santo, Brazil and successful treatment with praziquantel. Med Vet (UFRPE). 2018;12(1):17-21. doi: 10.26605/medvet-v12n1-2138.

37. Mitchell K, Kruth S. Immune-mediated hemolytic anemia and other regenerative anemias. In: Ettinger SJ, Feldman EC, editors. Textbook of veterinary internal medicine. 7 th ed. St. Louis: Else $\neg$ vier; 2010. p. 761-72.

38. Mayhew PD, Weisse CW. Treatment of pancreatitis-associated extrahepatic biliary tract obstruction by choledochal stenting in seven cats. J Small Anim Pract. 2008;49(3):133-8. doi: 10.1111/j.1748-5827.2007.00450.x.

39. Youn G, Waschak MJ, Kunkel KAR, Gerard PD. Outcome of elective cholecystectomy for the treatment of gallbladder disease in dogs. J Am Vet Med Assoc [Internet]. 2018 [citado 23 Maio 2021];252(8):970-5. Disponível em: https://pubmed.ncbi.nlm.nih.gov/29595398/

40. Benevides MPA. Laparoscopic cholecystectomy in dogs. PUBVET. 2021;15(7):1-14. doi:10.31533/pubvet.v15n07a868.1-14. 